\title{
Analysis of Performance Indices of Planar Parallel Manipulators
}

P. Abhilash and A. Chandrashekhar*

Mechanical Department, FST-IFHE, Hyderabad - 501503, Telangana, India; abhilashperu@gmail.com

\begin{abstract}
In this paper the performance analysis of one of the most commonly used parallel manipulator is been discussed. Performance analysis explains the difference performance factors of parallel manipulators like Condition number, Manipulability index, Transmission index, Minimum singular value, Stiffness index etc. In this Paper we are going to deal with indices like Transmission index, stiffness index and condition number. Three different structures are considered for the analysis. In each structure, for the entire dexterous workspace best postures of the manipulators are identified based on the performance measures.
\end{abstract}

Keywords: Condition Number, Parallel Manipulators, Performance Analysis, Stiffness Index, Transmission Index

\section{Introduction}

Manipulators have been used in most the fields now a days. The main classification of robots is series robot and parallel robots. Out of these series robots are simple and easy to use but parallel are little complex in building and analysis but they are more robust then series manipulators. In recent days research is going on usage of Parallel manipulators in many fields like even in medical fields. Parallel manipulators are classified based on their number of legs (the joints connecting the base pod and movable pod) as 3 legged, 4 legged, 5 legged, 6 legged etc and based on the joints as prismatic, revolute etc. In this paper I am working on a manipulator with 3 legs with RPR (Rotating-PrismaticRotating). This is a spatial manipulator. I developed an inverse kinematics for the manipulator and for out the performance. By plotting the performance indices at different structures we can select the best structure for that particular configuration. This method can help us to find the best manipulator for different environment and different usage.

\subsection{Planar 3RPR Parallel Manipulators}

\footnotetext{
*Author for correspondence

\section{Geometry of 3RPR Manipulator}

This planar 3 degrees-of-freedom parallel manipulator can also be built using prismatic joints. Here actually the $3 \mathrm{R}$ architecture of the legs is changed to an RPR architecture where the prismatic joint is the one that is actuated. This is shown in Figure 1, where, the distance between each of the Mis -which do not refer to motors here but to free pin joints-is set equal to unity. The assumption of symme-

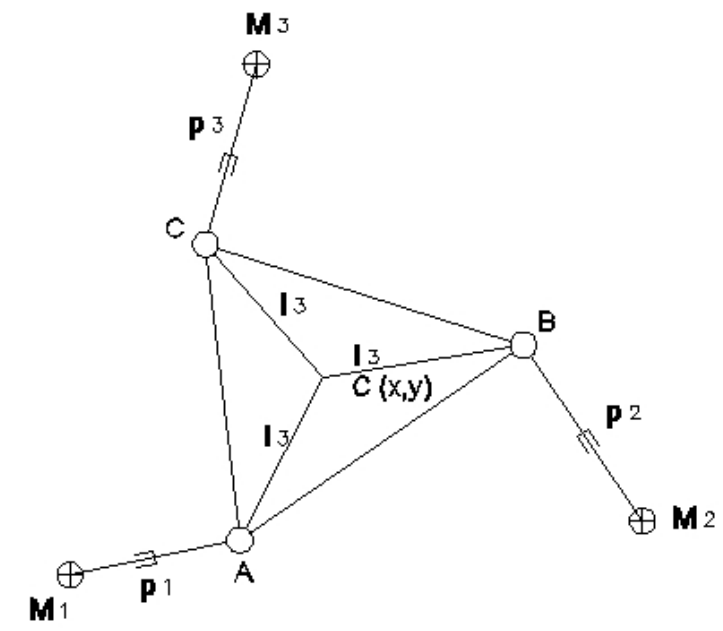

Figure 1. 3 RPR planar manipulator. 
try is also made here. The potential compensations of this manipulator over the one based on revolute actuators are easier kinematic equations and lesser mechanical interference. The applications for which this manipulator could be used are in medical field, manufacturing fields etc.

\section{Inverse Kinematics Analysis of 3RPR Manipulator}

Referring to Figure 1, we denote by $1_{3}$ the dimension of the gripper. by $\mathrm{p}_{\mathrm{i}}$ the length of the $i^{\text {th }}$ leg or actuator, and by $\left(x_{i}, y_{\mathrm{i}}\right)$ the coordinates of the point of attachment of the $i^{\text {th }}$ leg to the gripper. Moreover, the position of the point of the attachment of the $i$ th leg to the base is given by $\left(x_{\mathrm{oi}}, y_{\mathrm{oi}}\right)$, quantities that are given Equation (1). As in the case of the manipulator with revolute actuators, the Cartesian coordinates are given by the position of the centroid of the gripper $C(x, y)$ and by its orientation, defined here by angle $\emptyset$. We can then write

$$
\begin{aligned}
& x_{\mathrm{i}}=x-l_{3} \cos \emptyset_{i-x_{\text {oi }}} \quad i=1,2,3 \\
& y_{\mathrm{i}}=y-l_{3} \sin \emptyset_{i}-y_{\text {oir }}, \quad i=1,2,3
\end{aligned}
$$

where angles $\emptyset_{i}$ and the pairs $\left(x_{\mathrm{ni}} y_{\mathrm{ni}}\right)$ are given by

$$
\begin{gathered}
\emptyset_{1}=\emptyset+\pi / 6 \\
\emptyset_{2}=\emptyset+5 \pi / 6 \\
\emptyset_{3}=\emptyset-\pi / 2
\end{gathered}
$$

and

$$
\begin{aligned}
& \left\{x_{01}, x_{02}, x_{03}\right\}=\{0,1,1 / 2\} \\
& \left\{y_{01}, y_{02}, y_{03}\right\}=\{0,0, \sqrt{3} / 2\}
\end{aligned}
$$

The inverse kinematic problem, which has only one solution here, can then be solved using:

$$
p_{\mathrm{i}}=\sqrt{x_{i}^{2}+y_{i}^{2}}, \quad \mathrm{i}=1,2,3
$$

Therefore, given a certain position and orientation of the gripper, the required length of the actuators can be computed directly from Equation (5).

\section{Jacobian of a Planar 3RPR Manipulator}

The Jacobian matrix of the planar manipulator with prismatic actuators is defined similarly to the one of the manipulator with revolute actuators given in Equation (6). We define:

$$
\mathrm{J} \dot{\mathrm{c}}=\dot{\mathrm{p}}
$$

Where $\dot{\mathbf{c}}=[\dot{x}, \dot{y}, \emptyset]^{\mathrm{T}}$ is the vector of Cartesian velocities and

$\dot{\mathbf{p}}=\left[\dot{\mathrm{p}}_{1}, \dot{\mathrm{p}}_{2}, \dot{\mathrm{p}}_{3}\right]^{\mathrm{T}}$ is the vector of linear actuator rates.

The differentiation of Equation (5.5) leads to the Jacobian matrix

$$
\mathbf{J}=\left[\begin{array}{lll}
a 1 / p 1 & b 1 / p 1 & c 1 / p 1 \\
a 2 / p 2 & b 2 / p 2 & c 2 / p 2 \\
a 3 / p 3 & b 3 / p 3 & c 3 / p 3
\end{array}\right]
$$

Where

$$
\begin{gathered}
a_{\mathrm{i}}=x-x_{\mathrm{oi}}-l_{3} \cos \emptyset_{\mathrm{i}} \\
b_{\mathrm{i}}=y-y_{\mathrm{oi}}-l_{3} \sin \emptyset_{\mathrm{i}} \\
c_{\mathrm{i}}=\left(x-x_{\mathrm{oi}}\right) l_{3} \sin \emptyset_{\mathrm{i}}-\left(y-y_{\mathrm{oi}}\right) l_{3} \cos \emptyset_{\mathrm{I}}
\end{gathered}
$$

and the angles $\emptyset_{\mathrm{i}}$, for $i=1,2,3$, are defined as in Equation (2).

\section{Performance Indices of 3RPR Parallel Manipulator}

The three performance indices namely Transmission index, stiffness index and condition number are evaluated for the 3RPR manipulator. All the three indices are the functions of the Jacobian matrix.

\subsection{Jacobian Matrix}

In vector calculus, the Jacobian matrix is the matrix of all first-order partial derivatives of a vector-valued function. When the matrix is a square matrix, both the matrix and its determinant are referred to as the Jacobian in literature. Suppose $\mathrm{f}: \mathrm{Rn} \rightarrow \mathrm{Rm}$ is a function which takes as input the vector $x \in R n$ and produces as output the vector $\mathrm{f}(\mathrm{x}) \in \mathrm{Rm}$. Then the Jacobian matrix $\mathrm{J}$ of $\mathrm{f}$ is an $\mathrm{m} \times \mathrm{n}$ matrix usually defined and arranged as follows:

$$
J=\left[\frac{d f}{d x_{1}} \ldots \frac{d f}{d x_{n}}\right]
$$

Or, component-wise

$I_{i j}=\frac{d f_{i}}{d x_{j}}$ This matrix, whose entries are functions of $\mathrm{x}$, is also denoted by $\mathrm{D}_{\mathrm{f}}$ and $\mathrm{J}_{\mathrm{f}}$

\subsection{Condition Number}

When the determinant of the Jacobian is equal to zero, it means that the manipulator approaches singularities. However, the actual value of the determinant cannot be used as a practical measure of the degree of ill-conditioning. For this purpose it is convenient to use the condition 
number of the Jacobian. It is well known from the singular value decomposition theorem Condition number of nonsingular square matrix $\mathrm{M}$ defined by cond $\mathrm{M}=\|\mathrm{M}\|$. || $\mathrm{M} \|$ by convention, cond $(\mathrm{M})=\alpha$ if $\mathrm{M}$ singular.

\subsection{Transmission Index}

This is another criterion to know the performance whether it might be kinematic or dynamic. This index is important and it depends on the identity matrix combined with the norm movable pad matrix of the parallel manipulator in a matrix form.

\subsection{Stiffness Index}

The deformations or compliant displacements in the geometry of a body are caused due to application of load on the body. Stiffness can be defined as the capacity of a mechanical system to sustain loads without excessive changes of its geometry (Rivin, 1999). Moreover, the stiffness of a body can be defined as the amount of force that can be applied per unit of compliant displacement of the body (Nof, 1985), or the ratio of a steady force acting on a deformable elastic medium to the resulting displacement. Compliant displacements in a multibody robotic system allow for mechanical float of the end-effector relative to the fixed base. This produces negative effects on static and fatigue strength, efficiency (friction losses), accuracy, and dynamic stability (vibrations).

\section{Results}

\subsection{Condition Number}

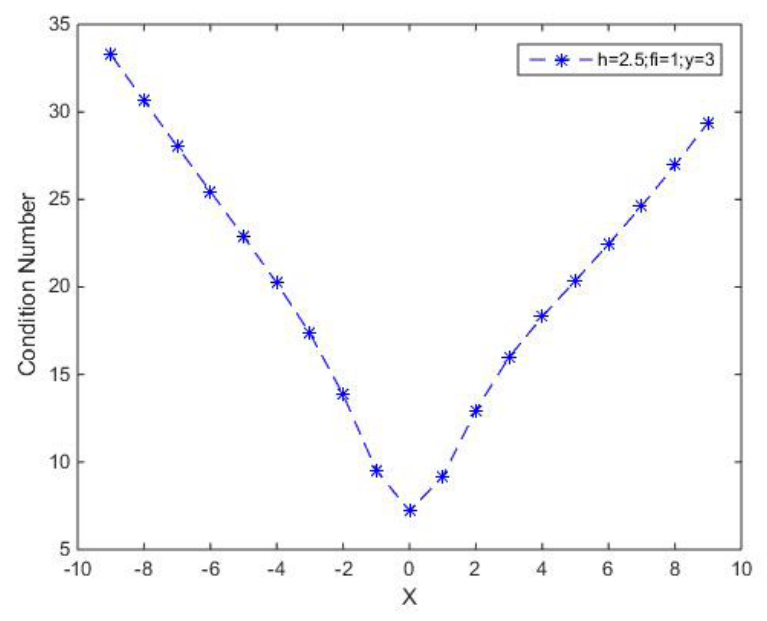

Figure 2. Condition number Vs reach of 3RPR manipulator for platform length of 2.5 and orientation of $1^{\circ}$.

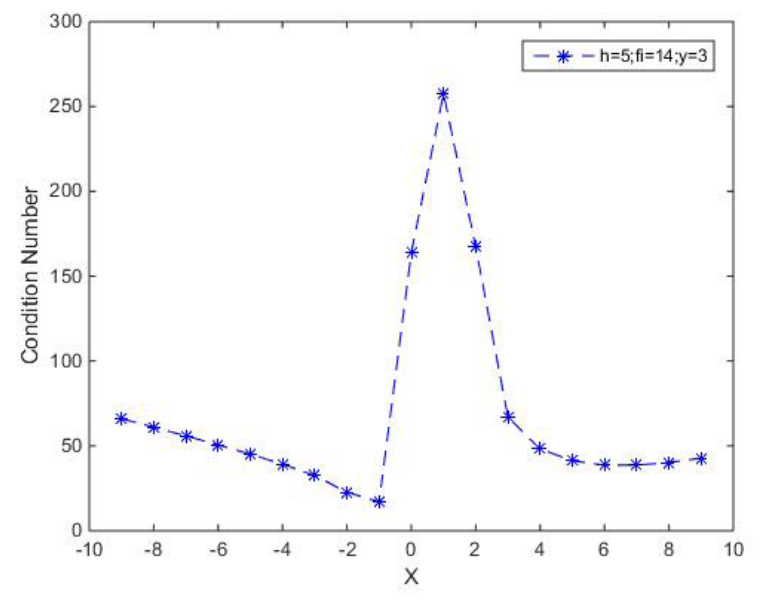

Figure 3. Condition number Vs reach of 3RPR manipulator for platform length of 5 and orientation of $14^{\circ}$.

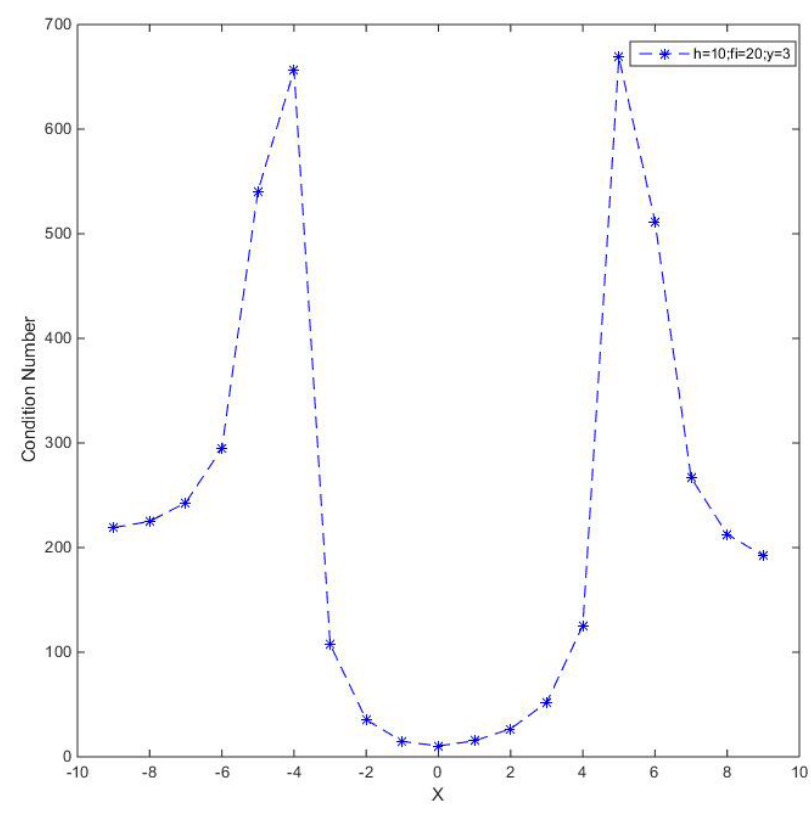

Figure 4. Condition number Vs reach of $3 R P R$ manipulator for platform length of 10 and orientation of $20^{\circ}$. 


\subsection{Transmission Index}

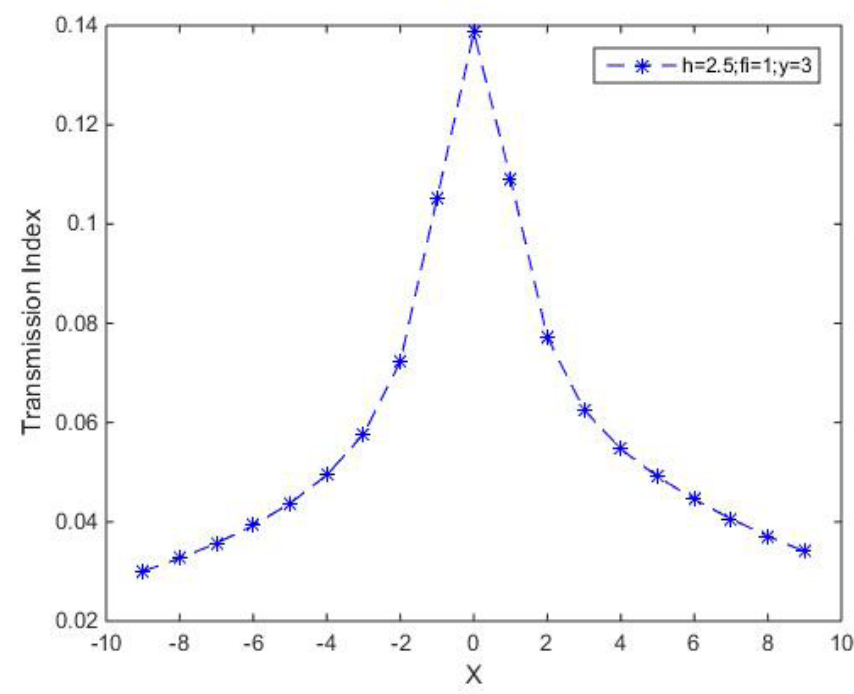

Figure 5. Transmission Index Vs reach of $3 R P R$ manipulator for platform length of 2.5 and orientation of $1^{\circ}$.

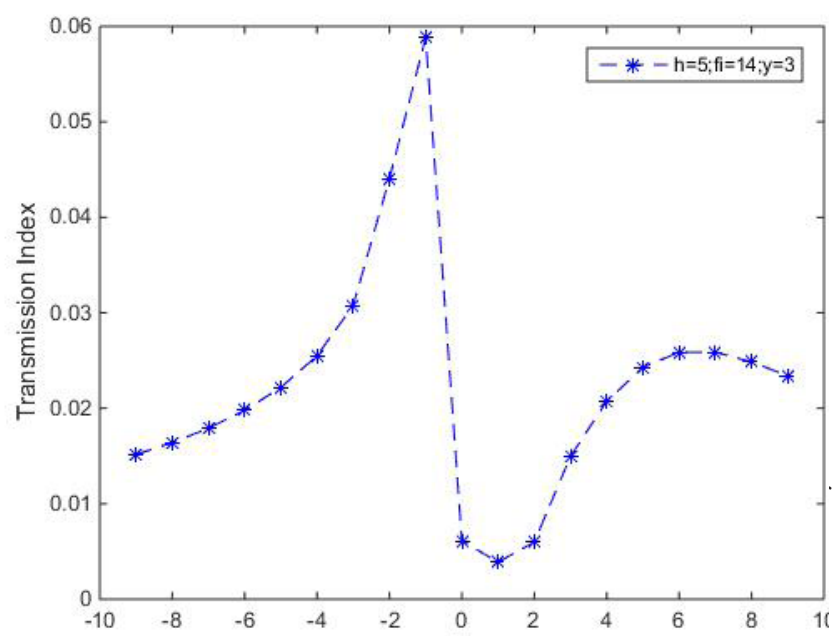

Figure 6. Transmission Index Vs reach of $3 R P R$ manipulator for platform length of 5 and orientation of $14^{\circ}$.

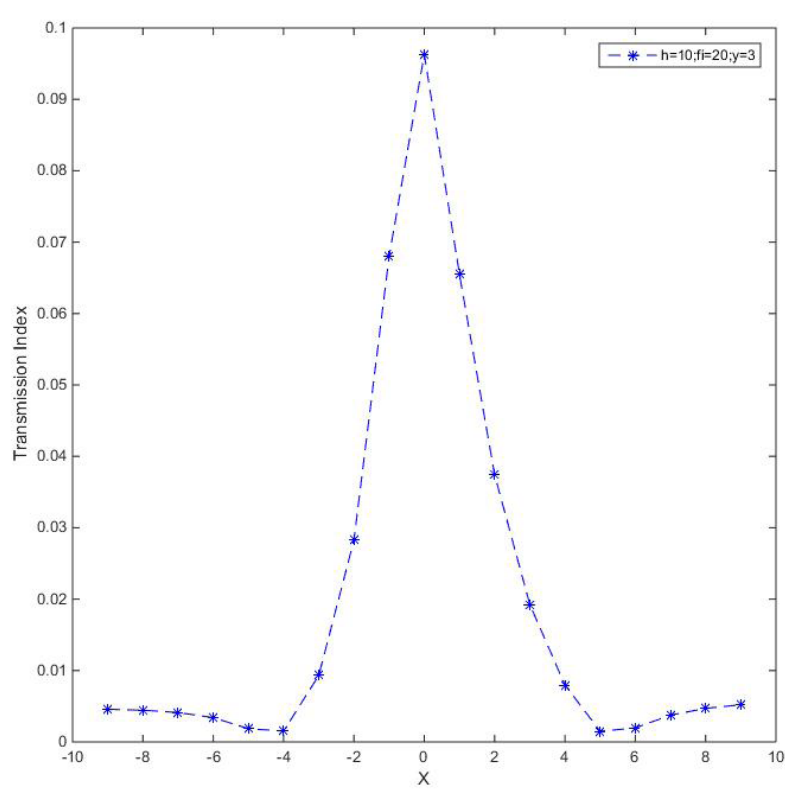

Figure 7. Transmission Index Vs reach of $3 R P R$ manipulator for platform length of 10 and orientation of $20^{\circ}$.

\subsection{Stiffness Index}

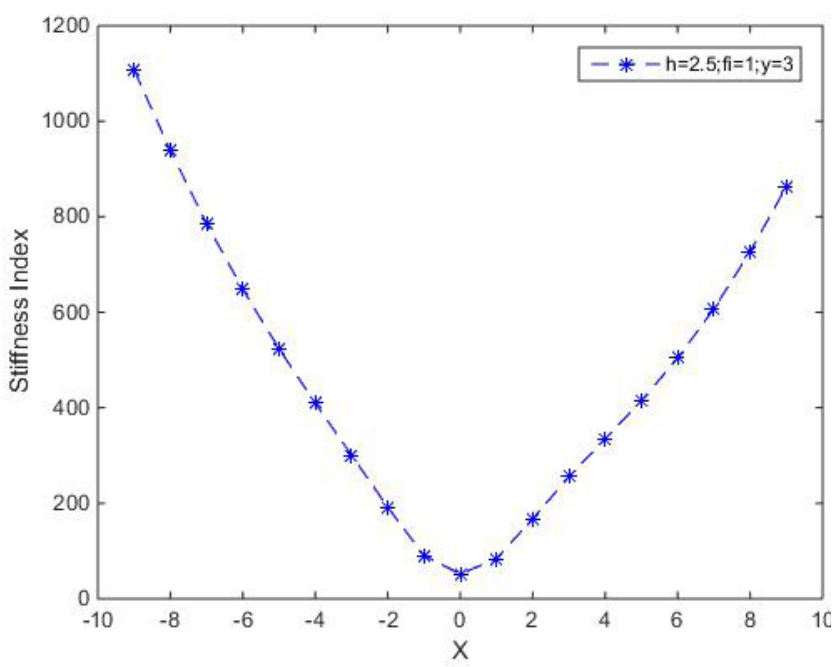

Figure 8. Stiffness Index Vs reach of 3RPR manipulator for platform length of 2.5 and orientation of $1^{\circ}$. 


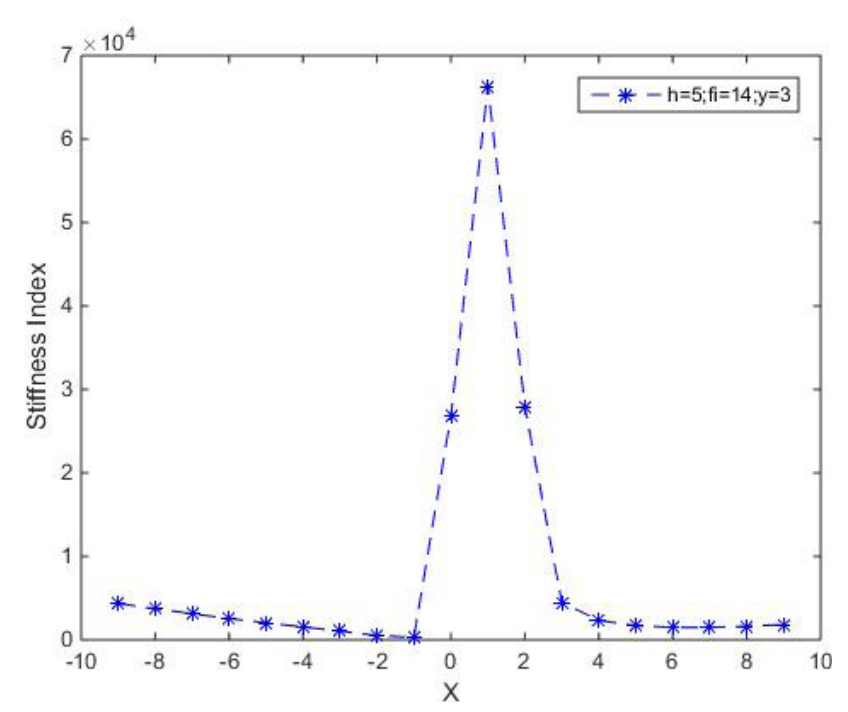

Figure 9. Stiffness Index Vs reach of 3RPR manipulator for platform length of 5 and orientation of $14^{\circ}$.

\section{Conclusion}

For the 3RPR planar parallel manipulator the base plate length is taken as unity. The length of the movable platform is expressed in terms of base plate length. Three different structures are considered for the analysis. In each structure, for the entire dexterous workspace best postures of the manipulators are identified based on the performance measures.

$\mathrm{h}=$ length of the movable platform

$x, y=$ the coordinates of the midpoint of the movable platform with respect to the frame attached to the first joint of the base plate.

$\mathrm{fi}=$ orientation angle of the platform

Structure I $: \mathrm{h}=2.5$

i.) when $f i=1^{\circ}$ and $y=3$ and $x=3$

Condition number $=33.985593$

Transmission index $=0.136826$

Stiffness Index $=1132.5042$

Structure II $: \mathrm{h}=5$

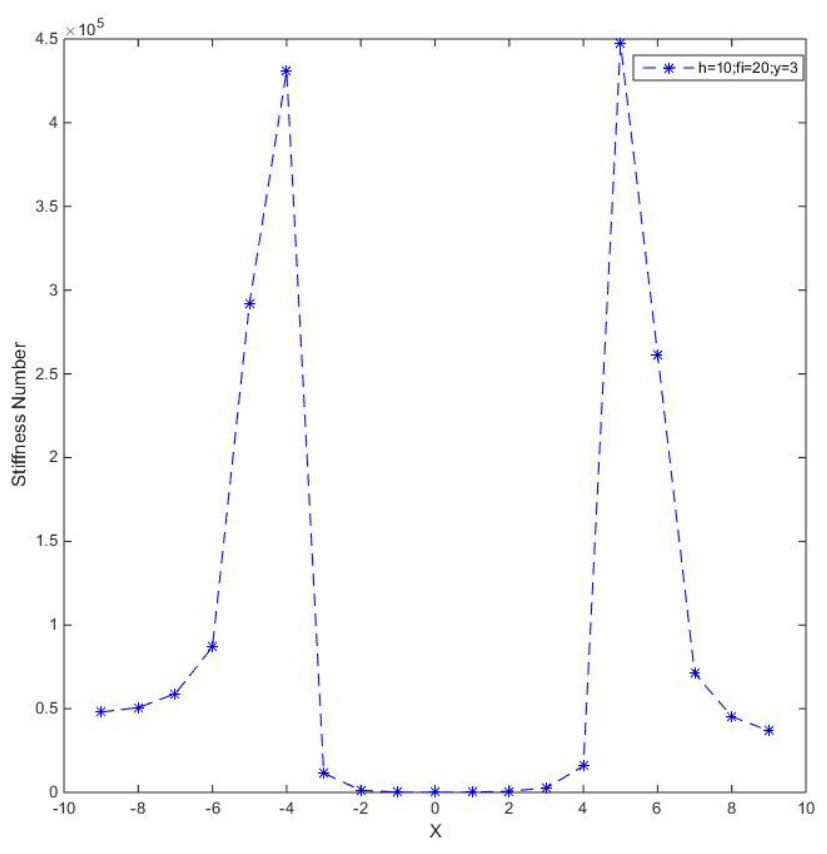

Figure 10. Stiffness Index Vs reach of 3RPR manipulator for platform length of 10 and orientation of $20^{\circ}$.

i.) when $\mathrm{fi}=14^{\circ}, \mathrm{y}=3$ and $\mathrm{x}=5$

Condition number $=261.9852$

Transmission index $=0.05982$

Stiffness Index $=66314.836237$

Structure III $: \mathrm{h}=10$

i.) when $\mathrm{fi}=20^{\circ}, \mathrm{y}=3$ and $\mathrm{x}=5$

Condition number $=781.55421$

Transmission index $=0.096826$

Stiffness Index $=430988.207100$

Structure III is the best one compared to other structures of the manipulator. Nearly isotropic configuration in the entire dexterous workspace is obtained for the posture with $f i=20^{\circ}$ and reach $(x=5$ and $y=3)$. Based on the Transmission measure the best posture of the manipulator is at $\mathrm{fi}=20^{\circ}$ and reach $(\mathrm{x}=5$ and $\mathrm{y}=3)$. The best posture based on the Stiffness index is at $\mathrm{fi}=15^{\circ}$ and reach $(x=5$ and $y=3)$. 


\section{References}

1. Rolland L, Rohitash C. Forward kinematics of the 3RPR planar parallel manipulators using real coded genetic algorithms. 24th International Symposium on Computer and Information Sciences; 2009. Crossref.

2. Chandra R, Zhang M, Rolland L. Solving the forward kinematics of the 3RPR planar parallel manipulator using a hybrid meta-heuristic paradigm. IEEE International Symposium on Computational Intelligence in Robotics and Automation - (CIRA); 2009. ISBN: 978-1-4244-4808-1.
3. Chandraa R, Rolland L. On solving the forward kinematics of 3RPR planar parallel manipulator using hybrid metaheuristics. Applied Mathematics and Computation. 2011 Jul; 217(22):8997-9008. Crossref.

4. Sahin S. Position equations of a 3RPR planar manipulator. Cite as: arXiv: 1508.01733 [cs.RO].

5. Zein $\mathrm{M}$, Wenger $\mathrm{P}$, Chablat $\mathrm{D}$. Non-singular assemblymode changing motions for 3-RPR parallel manipulators. Mechanism ad Machine Theory. 2008 Apr; 43(4):480-90. 